\title{
Laterally extended parametrectomy
}

\author{
Mihai Emil Căpîlna, MD1, Lászlo Ungár, MD², Alexandra Lavinia Cozlea, MD¹, Mihai Gheorghe, MD', \\ Mihai Stanca, MD¹, Balázs Lintner, $\mathrm{MD}^{3}$, Andreea Anamaria Moldovan, MD ${ }^{4}$

\begin{abstract}
${ }^{1}$ First Obstetrics and Gynecology Clinic, G. E. Palade University of Medicine, Pharmacy, Science and Technology, Târgu Mureș, Romania; ${ }^{2}$ Department of Gynecology, Duna Medical Center, ${ }^{3}$ Department of Gynecology, Semmelweis University, Budapest, Hungary; ${ }^{4}$ Department of Infectious Diseases and Epidemiology, County Hospital, Brașov, Romania
\end{abstract}

\section{Objective}

To describe the laterally extended parametrectomy (LEP) surgical technique, emphasizing the main challenges of the procedure.

\begin{abstract}
Methods
LEP was designed as a more radical surgical procedure aiming to remove the entire parametrial tissue from the pelvic sidewall. Its initial indications were for lymph node positive Stage Ib (current International Federation of Gynecology and Obstetrics 2018 Stage IIIc) and Stage Ilb cervical cancer. Currently, with most guidelines recommending definitive radiochemotherapy for these cases, initial LEP indications have become debatable. LEP is now mainly indicated for removing tumors involving the soft structures of the pelvic sidewall during a pelvic exenteration, aiming to obtain lateral free margins. This expands the lateral borders of the dissection to not only the medial surface of internal iliac vessels, but also to the true limits of the pelvic sidewall.
\end{abstract}

Results

During LEP, the parietal and visceral branches of the hypogastric vessels are divided at the entry and exit level of the pelvis. Consequently, the entire internal iliac system is excised, and no connective or lymphatic tissue remain on the pelvic sidewall. The main technical challenges of LEP are caused by the difficulty in ligating large caliber vessels (internal iliac artery and vein) and the variable anatomic distribution of pelvic sidewall veins.

\section{Conclusion}

LEP is a feasible technique for removing pelvic sidewall recurrences, aiming to obtain surgical free margins.

Keywords: Parametrectomy; Cervical cancer; Recurrences; Excision margins

The laterally extended parametrectomy (LEP) was designed as a more radical surgical procedure aiming to remove the entire parametrial tissue from the pelvic sidewall. Its initial indications were lymph node-positive stage lb (current International Federation of Gynecology and Obstetrics 2018 stage IIIc) and stage Illb cervical cancer $[1,2]$.

Nowadays, when most guidelines recommend definitive radiochemotherapy for these patients, the initial LEP indications are debatable $[3,4]$. LEP's main indication is to remove tumors involving the soft structures of the pelvic sidewall during a pelvic exenteration [5], aiming to obtain lateral free margins. LEP expands the lateral borders of the dissection not only to the medial surface of internal iliac vessels but to the true limits of the pelvic sidewall [2].

During LEP, together with the visceral branches of hypogas- tric vessels, all the parietal branches are also divided at the level where the vessels leave or enter into the pelvis (Fig. 1).

Received: 2021.03.18. Revised: 2021.04.21. Accepted: 2021.05.04. Corresponding author: Mihai Stanca, MD

University of Medicine, Pharmacy, Science and Technology G.E. Palade of Târgu Mures, 38 Gheorghe Marinescu Street, Târgu

Mures 540142, Romania

E-mail: doctorstanca@gmail.com

https://orcid.org/0000-0002-2535-509X

Articles published in Obstet Gynecol Sci are open-access, distributed under the terms of the Creative Commons Attribution Non-Commercial License (http://creativecommons. org/licenses/by-nc/3.0/) which permits unrestricted non-commercial use, distribution, and reproduction in any medium, provided the original work is properly cited.

Copyright $\odot 2021$ Korean Society of Obstetrics and Gynecology 


\section{Obstetrics \& Gynecology Science}

Mihai Emil Căpîlna, et al. Laterally extended parametrectomy

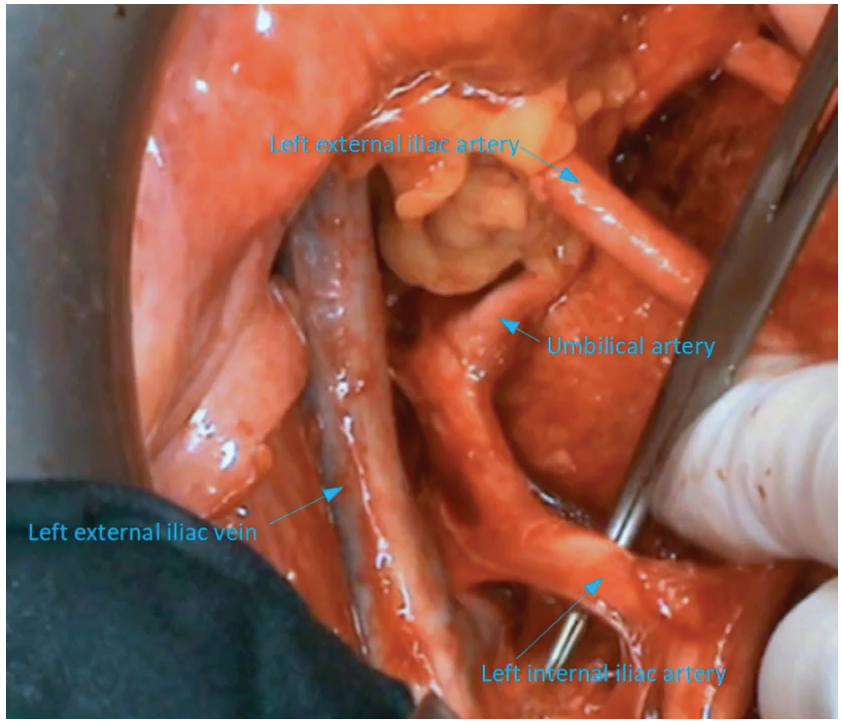

Fig. 1. Left pelvic side wall after laterally extended parametrectomy in another patient.

Consequently, the entire internal iliac system is excised, and no connective or lymphatic tissue remains on the pelvic sidewall [1].

The main technical challenge of LEP is caused by the difficulty to ligate large-caliber vessels (internal iliac artery and vein) and by the variable anatomic distribution of pelvic sidewall veins $[2,6]$. LEP is a feasible technique for removing pelvic sidewall recurrences, aiming to obtain surgical-free margins.

\section{Conflict of interest}

No potential conflict of interest relevant to this article was reported.

\section{Ethical approval}

The study was conducted under the approval of the appropriate Ethical Committee (ethical approval code: 34535 ) and written informed consent was obtained from the subject. The study was performed in accordance with the principles of the Declaration of Helsinki.

\section{Patient consent}

Written informed consent and the use of images from patients are not required for the publication.

\section{Funding information}

None.

\section{Acknowledgments}

To Andris Barabás, for his contribution with video editing. A different video of the same technique was presented at the 2020 xDigital Annual Global Meeting of the International Gynecologic Cancer Society.

\section{Video clip}

Video can be found with this article online at https://doi. org/10.5468/ogs.21103.

\section{References}

1. Ungár $L$, Pálfalvi L. Surgical treatment of lymph node metastases in stage IB cervical cancer: the laterally extended parametrectomy (LEP) procedure. Int J Gynecol Cancer 2003;13:647-51.

2. Pálfalvi L, Ungár L. Laterally extended parametrectomy (LEP), the technique for radical pelvic side wall dissection: feasibility, technique and results. Int J Gynecol Cancer 2003;13:914-7.

3. Cibula D, Dostalek L, Hillemanns P, Scambia G, Jarkovsky J, Persson J, et al. Completion of radical hysterectomy does not improve survival of patients with cervical cancer and intraoperatively detected lymph node involvement: ABRAX international retrospective cohort study. Eur J Cancer 2021;143:88-100.

4. Stanca M, Căpîlna ME. Prognostic factors associated with 5-year overall survival in cervical cancer patients treated with radical hysterectomy followed by adjuvant concurrent chemoradiation therapy at a tertiary 


\section{Obstetrics \& Gynecology Science}

Vol. 64, No. 5, 2021

care center in Eastern Europe. Diagnostics (Basel) 2021;11:570.

5. Căpîlna ME, Moldovan B, Szabo B. Pelvic exenteration-our initial experience in 15 cases. Eur J Gynaecol Oncol 2015;36:142-5.
6. Căpîlna ME, Szabo B, Rusu SC, Becsi J, Moldovan B, Neagoe RM, et al. Anatomical variations of the obturator veins and their surgical implications. Eur J Gynaecol Oncol 2017;38:263-5. 